\title{
Grand Challenges in Soft Matter Physics
}

\author{
Jasper van der Gucht* \\ Physical Chemistry and Soft Matter, Wageningen University, Wageningen, Netherlands
}

Keywords: soft matter physics, self-assembly, non-equilibrium physics, active matter, polymers, colloids

\section{OPEN ACCESS}

Edited and reviewed by: Alex Hansen,

Norwegian University of Science and Technology, Norway

*Correspondence:

Jasper van der Gucht jasper.vandergucht@wur.nl

Specialty section:

This article was submitted to Soft Matter Physics,

a section of the journal Frontiers in Physics

Received: 26 June 2018 Accepted: 23 July 2018 Published: 22 August 2018

Citation:

van der Gucht J (2018) Grand Challenges in Soft Matter Physics. Front. Phys. 6:87.

doi: 10.3389/fphy.2018.00087
As its name implies, soft matter science deals with materials that are easily deformed. These materials, which include polymers, gels, colloids, emulsions, foams, surfactant assemblies, liquid crystals, granular materials, and many biological materials, have in common that they are organized on mesoscopic length scales, with structural features that are much larger than an atom, but much smaller than the overall size of the material. The large size of the basic structural units and the relatively weak interactions that hold them together are responsible for the characteristic softness of these materials ${ }^{1}$, but they also lead to many other distinct features of soft materials [1], such as sensitivity toward thermal fluctuations and external stimuli and a slow response with long relaxation times, often resulting in non-trivial flow behavior and arrest in non-equilibrium states. These features make soft matter problems challenging. In hard condensed matter physics, it is often possible to accurately predict material properties based on the interactions between the individual atoms, which are typically organized on a regular crystalline lattice. For soft matter systems, with their intrinsically heterogeneous structure, complex interactions across different length scales, and slow dynamics, this is much more difficult. The subtle interplay between interactions and thermal fluctuations can lead to complex emergent behavior, such as spontaneous pattern formation, self-assembly, and a large response to small external stimuli.

Because of the wide range of materials and systems that can be classified as soft matter, soft matter science is an inherently interdisciplinary field, in which physics, chemistry, materials science, biology, nanotechnology, and engineering come together. For a field that is so broad in scope, it is impossible to do justice to the entire range of outstanding problems or even to identify two or three key challenges. For this reason, I will only highlight a small (and highly personal) selection of current challenges in the field. The interdisciplinary nature of the field will be evident from these examples.

\section{MOLECULAR SELF-ASSEMBLY}

One of the hallmarks of soft matter systems is the spontaneous formation of mesoscopic structures from smaller building blocks, in a process that we call self-assembly [2]. Probably the simplest example of self-assembly is that of amphiphilic molecules (or surfactants) into micelles or vesicles. Much more intricate examples can be found in biology, where self-assembly leads to complex, multi-component structures, such as viruses, hierarchically organized collagen fibers, the intracellular cytoskeleton, or DNA-enzyme complexes responsible for replication of DNA. These biological examples have inspired scientists all over the world to create increasingly complex molecules that can self-assemble into spherical or cylindrical micelles, vesicles, fibers, ribbons, supramolecular gels, or more complex hierarchical structures [3]. However, despite the impressive

\footnotetext{
${ }^{1}$ This is easily seen from dimension analysis: the elastic moduli that characterize the rigidity of a material have units energy/volume, so that building blocks with comparable interaction energy, but much larger volume must lead to moduli that are orders of magnitude smaller than for atomic or molecular crystals.
} 
progress in the last few decades, these synthetic self-assembled structures are still far away from the complexity found in nature.

The central idea in designing synthetic self-assembling systems is that the final structure and function can be preprogrammed into the shape of the individual building blocks and the interactions between them. For simple cases, the final structure corresponds to the thermodynamic equilibrium situation, so that the outcome of the self-assembly process can in principle be predicted theoretically by models based on free energy minimization. However in more complex situations, for example when multiple species interact orthogonally within the same system or when different self-assembly pathways compete with one another, the free energy landscape may exhibit many local minima in which the assembling structures can become kinetically trapped. To find rational design principles for creating hierarchical self-assembled structures, we must unravel the complexity that emerges when orthogonal interactions and kinetic pathways interplay. For this, physics-based modeling is needed, together with novel tools that can probe the fine details of the self-assembly process [4].

\section{COLLOIDAL PHASE BEHAVIOR}

An alternative strategy to form materials from the bottom up, is by starting with nanometer-sized colloidal building blocks. Colloidal particles can be synthesized in many sizes and shapes, and the interactions between them can be tuned, for example by varying the surface charge, by changing the $\mathrm{pH}$ or salt concentration, by adding polymeric additives, or by decorating the particles with polymers, specific ligands, or complementary DNA strands. This can lead to self-organization into a large variety of structures and phases, such as colloidal liquids, crystals, liquid crystals, and glasses, to a large extent mimicking the phase behavior of atoms and molecules. Since colloids are big and slow enough to be followed individually using an optical microscope, they can be used to study fundamental physics problems at the individual particle level, such as nucleation, melting, and the properties of crystal defects [5]. More practically, carefully designed colloidal building blocks may once become the "atoms" and "molecules" of future materials, with potentially very interesting optical, mechanical, and catalytic properties. Before realizing this, however, we must overcome challenges that are very similar to those for molecular self-assembling systems: how can we program the desired structure into the individual building blocks? Again, answering this question relies on a combined approach, in which computer simulations are needed to rationalize and guide the experimental design. However, compared to self-organization of atoms and molecules, colloidal self-organization poses a number of additional challenges: (i) Contrary to atomic interactions, most colloidal interaction potentials are isotropic. This limits the range of lattice structures that can be formed to those with a simple unit cell, such as face-centered cubic (fcc) and bodycentered cubic (bcc) crystals. To obtain other crystal symmetries, particles with anisotropic shape or interaction potential or with a small number of sticky patches are needed. Several methods to prepare such particles have been described in recent years [6], but the number of successfully assembled crystal structures remains limited. (ii) Non-equilibrium states are even more prominent for colloids than for molecular self-assembly. Gelation of particles can arrest the particles in a non-equilibrium aggregated state, completely suppressing crystallization. Especially for relatively large attractive particles, gelation is very difficult to avoid. (iii) While in simulations virtually any interaction potential can be implemented, it is difficult to design colloidal building blocks with a precisely specified interaction potential between them. Mapping simulation results onto experimental systems therefore remains an important challenge.

\section{FLOW AND RHEOLOGY; GLASSES AND JAMMING}

As a result of the weak interactions and the slow relaxation processes, soft matter systems have non-trivial flow behavior, characterized by a viscoelastic and often non-linear response. One of the challenges in the field is to relate the macroscopic rheological signature to the microscopic structure and interactions. While this has been achieved successfully for some systems (for example, the tube model for polymer dynamics), in many cases a microscopic understanding is still missing. One class of materials that remains particularly poorly understood is that of glasses. Upon cooling, the viscosity of a glass-forming liquid can increase by many orders of magnitude. At the glass transition, the viscosity has become so high that the sample appears macroscopically as a solid; yet microscopically it still has the structure of a liquid. Polymers are particularly good glass formers, but when cooled sufficiently fast, virtually any liquid forms a glass. Colloidal suspensions can also undergo a glass transition, in this case upon increasing the volume fraction of particles. While the physical phenomena that accompany the glass transition are well-described, the fundamental microscopic mechanism that underlies it remains one of the outstanding (and heavily debated) problems in (soft) condensed matter physics [7]. What transformation occurs when a liquid becomes glass? Is there a thermodynamic glass transition? And why do some liquids show a gradual, Arrhenius-type increase in viscosity with decreasing temperature, while others show a much steeper increase? How is the colloidal glass transition related to that of molecular glass formers? The rapidly increasing relaxation times close to the glass transition make answering these questions extremely difficult, both experimentally and in simulations.

Apart from the glass transition, also the properties of the glass itself need further study. Under stress, glasses may creep or yield. The dynamics that govern these processes are highly heterogeneous and characterized by soft spots where the majority of particle rearrangements occur. However, identifying these soft spots and relating them to structural features remains a major challenge: while for a crystal it is obvious where the defects are, the concept of a defect becomes rather ill-defined in an amorphous solid. Colloidal glasses may be the ideal model system to study the physics of glasses and supercooled liquids, since 
the soft spots and heterogeneous particle rearrangements can be studied at the individual particle level [8].

When the particles grow bigger in size so that Brownian motion becomes negligible, we enter the area of granular materials. The "grains" may be solid particles (as in sand), liquid droplets (as in emulsions), or gas bubbles (as in foams). Granular materials have a strongly non-linear response to external forces. They can be solid-like when left undisturbed, but start to flow in response to a moderate external force. Much of the flow behavior of granular matter can be captured by phenomenological models, such as the Herschel-Bulkley model for yield-stress emulsions or scaling approaches based on jamming arguments [9]. However, there is at present no microscopic model that explains the rich flow behavior of granular suspensions and emulsions or that can relate the empirical constants in the phenomenological models to microscopic quantities.

\section{ACTIVE MATTER AND BIOLOGICAL SOFT MATTER}

The examples above highlight the importance of non-equilibrium aspects in soft matter science, due to kinetically arrested states or external driving forces. A special class of materials is that of active matter. These are systems that are intrinsically out of equilibrium, because the particles continuously consume energy that is used for their active movement or to exert mechanical forces. The interplay between a large number of these active particles can lead to very complex patterns of collective motion and self-organized structures. Many examples of such patterns can be seen in biology: in flocks of birds, in schools of fish, or in colonies of bacteria, but also at a smaller scale, where actively growing and shrinking microtubules and actin filaments control the structure and dynamics of living cells, together with motor proteins that exert forces on these filaments. Active matter systems display very rich physics, with features not observed in passive systems, such as internally generated flow patterns

\section{REFERENCES}

1. Nagel SR. Experimental soft-matter science. Rev Mod Phys. (2017) 89:025002. doi: 10.1103/RevModPhys.89.025002

2. Whitesides G, Grzybowski B. Self-assembly at all scales. Science (2002) 295:2418-21. doi: 10.1126/science.1070821

3. Capito RM, Azevedo HS, Velichko YS, Mata A, Stupp SI. Self-assembly of large and small molecules into hierarchically ordered sacs and membranes. Science (2008) 319:1812-6. doi: 10.1126/science.1154586

4. Cingil HE, Boz EB, Biondaro G, de Vries R, Stuart MAC, Kraft DJ, et al. Illuminating the reaction pathways of viromimetic assembly. J Am Chem Soc. (2017) 139:4962-8. doi: 10.1021/jacs.7b01401

5. Poon WCK. Colloids as big atoms: the genesis of a paradigm. J Phys A Math Theor. (2016) 49:401001. doi: 10.1088/1751-8113/49/40/401001

6. Chen Q, Bae SC, Granick S. Directed self-assembly of a colloidal kagome lattice. Nature (2011) 469:381-4. doi: 10.1038/nature09713

7. Stillinger FH, Debenedetti PG. Glass transition thermodynamics and kinetics. Annu Rev Condens Matter Phys. (2013) 4:263-85. doi: 10.1146/annurev-conmatphys-030212-184329

8. Weeks E, Crocker J, Levitt A, Schofield A, Weitz D. Three-dimensional direct imaging of structural relaxation near the colloidal glass transition. Science (2000) 287:627-31. doi: 10.1126/science.287.54 53.627 and mechanical stresses, large-scale collective motion, active force generation, unusual fluctuations, non-equilibrium phase transitions, and sustained oscillations. Since active matter is inherently far from equilibrium, the statistical physics framework commonly used to describe soft matter systems breaks down, and new theory is needed. Given the enormous complexity of biological matter, a general theoretical description of these phenomena is still far away. Nevertheless, it may be possible to find rules and principles that govern the complex behavior of active matter [10]. Well-controlled experiments with model systems that are simpler than the biological examples, such as active colloids, active liquid crystals or active gels will be essential for making progress $[11,12]$.

\section{DESIGNER SOFT MATTER}

As is clear from the discussion so far, a common thread in soft matter physics is to find microscopic explanations for macroscopically observed behavior. The inverse problemdesigning a material with a specific desired property-is obviously much more difficult. Yet, it would be highly desirable to be able to predict what kind of polymers one should make to achieve a certain mechanical response, to know what colloidal particles to make to realize a crystal with certain optical properties, or to predict the shape that particles should have to achieve a granular packing with a desired rheological response. Clearly this requires an approach in which simulations, theory and experiments are combined. With the increase in computation power, the development of novel experimental tools, and new algorithms for analyzing large data sets, this dream may soon be within reach.

\section{AUTHOR CONTRIBUTIONS}

The author confirms being the sole contributor of this work and approved it for publication.

9. Bonn D, Denn MM, Berthier L, Divoux T, Manneville S. Yield stress materials in soft condensed matter. Rev Modern Phys. (2017) 89:035005. doi: 10.1103/RevModPhys.89.035005

10. Marchetti MC, Joanny JF, Ramaswamy S, Liverpool TB, Prost J, Rao M, et al. Hydrodynamics of soft active matter. Rev Modern Phys. (2013) 85:1143-89. doi: 10.1103/RevModPhys.85.1143

11. Palacci J, Sacanna S, Steinberg AP, Pine DJ, Chaikin PM. Living crystals of light-activated colloidal surfers. Science (2013) 339:936-40. doi: $10.1126 /$ science. 1230020

12. Sanchez T, Chen DTN, DeCamp SJ, Heymann M, Dogic Z. Spontaneous motion in hierarchically assembled active matter. Nature (2012) 491:431-5. doi: $10.1038 /$ nature 11591

Conflict of Interest Statement: The author declares that the research was conducted in the absence of any commercial or financial relationships that could be construed as a potential conflict of interest.

Copyright (C) 2018 van der Gucht. This is an open-access article distributed under the terms of the Creative Commons Attribution License (CC BY). The use, distribution or reproduction in other forums is permitted, provided the original author(s) and the copyright owner(s) are credited and that the original publication in this journal is cited, in accordance with accepted academic practice. No use, distribution or reproduction is permitted which does not comply with these terms. 